\title{
REVISIÓN
}

\section{Aplicación de los sistemas TOAST y CCS en el diagnóstico de accidente cerebrovascular isquémico}

\author{
Facundo Martín,* María Emilia Tarducci,** Sandra M Tabares,* Juan José Martín,*** Adela Sembaj* \\ * Cátedra de Bioquímica y Biología Molecular. Facultad de Ciencias Médicas, Universidad Nacional de Córdoba. \\ ** Servicio de Neurología, Sanatorio Allende, Nueva Córdoba. \\ *** Facultad de Medicina, Universidad Católica de Córdoba.
}

\begin{abstract}
RESUMEN
Introducción: Nos propusimos comparar la eficiencia diagnóstica de los sistemas de clasificación TOAST y CCS en pacientes con ACVi que asisten al Sanatorio Allende, Córdoba. Material y métodos: Se aplicaron los sistemas de clasificación TOAST y CCS a 100 pacientes que asistieron al Servicio de Neurología del Sanatorio Allende, en forma retrospectiva. Se analizó la eficiencia diagnóstica mediante el índice kappa. Resultados: Según CCS, tres (3\%) de los pacientes fueron de etiología no determinada, desconocida; 10 (10\%) de etiología indeterminada, desconocida/otras causas criptogénicas; dos ( $2 \%$ ) no determinada, no conocida/ embólica criptogénica; y cinco (5\%) de etiología no determinada, no conocida/evaluación incompleta; dando un total de 20 (20\%). Según TOAST, 39 (39\%) se ubicaron en la categoría de etiología indeterminada. Se precisó un acuerdo diagnóstico muy bueno entre los subtipos asignados por TOAST y el CCS $(K=0.81)$. Para los principales subtipos, la concordancia también fue muy buena; para aterosclerosis de grandes vasos $(\mathrm{k}=0.898)$, oclusión de arterias pequeñas $(\mathrm{k}=0.98)$, cardioembolia $(\mathrm{k}=0.95)$, otras causas $(\mathrm{k}=0.88)$. Se observó acuerdo moderado para la categoría indeterminada $(\mathrm{k}=0.564)$. Conclusiones: El sistema CCS mostró ser más eficiente para definir la etiología del ACVi en comparación con el TOAST; dado que es un algoritmo web aumenta su confiabilidad entre evaluadores.
\end{abstract}

Palabras clave: Accidente cerebrovascular isquémico (ACVi), diagnóstico, CCS, TOAST.

\begin{abstract}
Introduction: We propose to compare the diagnostic efficiency of the TOAST and the CCS classification systems in patients with stroke who attend at the Sanatorium Allende, Cordoba. Material and methods: The TOAST and CCS classification systems were applied to 100 patients who were admitted to the Neurology Service of the Sanatorium Allende, from the data of the clinical records in a retrospective way. To the diagnostic efficiency of both types of classification was analyzed by kappa index. Results: According to CCS 3 (3\%) of the patients were undetermined etiology, 10 (10\%) other cryptogenic causes, 2 (2\%) not determined known / cryptogenic embolic and 5 (5\%) undetermined etiology/incomplete evaluation, totaling 20 (20\%); according to TOAST 39 (39\%) of the patients were of indeterminate etiology. There was a very good diagnostic agreement between the subtypes assigned by TOAST and the CCS $(K=0.81)$. For the main subtypes the agreement was also very good: large vessel atherosclerosis $(k=0.898)$, small artery occlusion $(k=0.98)$, cardioembolism $(k=0.95)$, others causes $(k=0.88)$. Moderate agreement was observed for the indeterminate category $(k$ $=0.564)$. Conclusion: The CCS system showed to be more efficient to determine the etiology of the ICVA compared to the TOAST. Also, is it a web algorithm increases the reliability among evaluators.
\end{abstract}

Keywords: Isquemic cerebrovascular accident (ICVA), diagnosis, CCS, TOAST.

\section{INTRODUCCIÓN}

Identificar la causa del accidente cerebrovascular isquémico (ACVi) es importante en la práctica clínica para orientar las decisiones de tratamiento y pronóstico en los pacientes. También lo es la designación de subtipos etiológicos de ACVi como elementos básicos

\footnotetext{
Abreviaturas:

ACVi = Accidente cerebrovascular isquémico.

TOAST = Trial of Org 10172 in Acute Stroke Registry.

CCS = Causative Classification of Stroke System.
}

en la investigación clínica de las enfermedades cerebrovasculares, dada la heterogeneidad de los mecanismos biológicos subyacentes que lo desencadenan. Por tanto, es valioso tener un sistema de clasificación fácil de replicar, en el que todos los términos usados sean suficientemente claros y que se puedan utilizar de forma indistinta por diferentes investigadores. ${ }^{1} \mathrm{La}$ mayoría de los sistemas o escalas que se utilizan en la investigación clínica del ACVi no logran alcanzar una excelente fiabilidad; esto produce errores de clasificación en la investigación, además de erosionar la eficiencia y la potencia de los estudios clínicos. ${ }^{2}$ 
El sistema de clasificación del tratamiento de ACVi agudo, Trial of Org 10172 in Acute Stroke Registry (TOAST), fue desarrollado para mejorar la estandarización de la asignación de subtipos para estudios clínicos aleatorizados y multicéntricos. ${ }^{3}$ Sin embargo, el TOAST se aplica en estudios epidemiológicos y genéticos de asociación para los que no fue originalmente diseñado, ${ }^{1}$ pero la amplia utilización del sistema TOAST y los avances en la tecnología de diagnóstico han permitido identificar algunos defectos. Como en pacientes con múltiples y potenciales mecanismos desencadenantes de un ACVi, mecanismos que el sistema no reconoce y son clasificados como de causa indeterminada, como consecuencia los pacientes terminan siendo investigados de forma incompleta. Este subgrupo a menudo se excluye del análisis, lo que conduce a un uso poco óptimo de la información disponible. ${ }^{4}$

En el 2007, Hy A y colaboradores presentaron un sistema de clasificación denominado Causative Classification of Stroke System (CCS) para superar el déficits del TOAST; este nuevo sistema es un algoritmo basado en evidencia y diseñado para determinar la etiología más probable en presencia de múltiples mecanismos. ${ }^{2,3}$ El CCS tiene la capacidad de refinar la asignación de subtipos y mejorar el uso de los datos en los estudios clínicos multicéntricos en pacientes con ACVi. ${ }^{5}$ Además, el sistema asigna a pacientes con el subtipo más probable sobre la base de las patologías cardiacas y vasculares; también asigna patrones clínicos y patrones de imagen que se observan y asocian con mayor frecuencia a mecanismos particulares de ACVi. Se espera que esto reduzca el número de pacientes clasificados como no determinados, especialmente cuando se debe a más de una patología potencialmente involucrada. ${ }^{6}$

Los errores de clasificación generalmente surgen de tres fuentes: los datos resumidos de los pacientes, el evaluador y el sistema de entrada. Los errores relacionados con los datos incluyen ambigüedades en las historias clínicas, incoherencias obtenidas mediante pruebas similares (tomografía computarizada y resonancia magnética) y/o la falta de datos; estos errores son críticos para diferenciar subtipos (lo que puede inducir a los evaluadores a utilizar su mejor estimación). El sistema de clasificación CCS ofrece una serie de características para evitar que los usuarios introduzcan datos inconsistentes. ${ }^{5,6}$

En nuestro medio, el sistema diagnóstico CCS se utiliza con poca frecuencia en los servicios de neurología, de manera que contamos con pocos datos respecto al porcentaje de casos clínicos asignados según los subtipos de CCS y su comparación con TOAST. Por esa razón, nos hemos propuesto aplicar los sistemas de clasificación CCS y TOAST a una cohorte de pacientes con accidente cerebrovascular isquémico asistidos en el Sanatorio Allende; para, a partir de los datos de las historias clínicas en forma retrospectiva, describir las frecuencias de los subtipos de accidente cerebrovascular y comparar la eficiencia diagnóstica de ambos tipos de clasificación.

\section{Material Y MÉTODOS}

Población de estudio: se analizaron de forma retrospectiva los datos clínicos de pacientes con ACVi que asistieron consecutivamente al Servicio de Neurología del Sanatorio Allende entre marzo de 2017 a marzo de 2018. Este proyecto fue evaluado por el Comité de Ética y la institución autorizó el uso de los datos clínicos para la investigación (Sanatorio Allende, 08/09/2016).

El ACVi se definió como un síndrome clínico caracterizado por su rápido desarrollo, que presenta síntomas neurológicos focales (a veces globales) y coma de pérdida de función, que dura más de una hora sin otra causa aparente más que de origen vascular, y que además cuenta con evidencia radiológica de un infarto agudo observada con técnicas de neuroimágenes. Se realizó una revisión diaria de los registros de ingresos hospitalarios y solicitudes de consultas a la unidad especializada (Unidad de Ataque Cerebrovascular), y una a la guardia para determinar algún nuevo ingreso de pacientes hospitalizados, así como la cantidad de eventos en el Sanatorio. El Servicio de Neurología del Sanatorio Allende procede según las recomendaciones para el manejo del paciente con ACVi indicadas en J Stroke 2018 (AHA/ASA Stroke Early Management Guidelines). ${ }^{7}$

Se definió hipertensión en pacientes con tratamiento antihipertensivo y en aquéllos que presentaron de forma persistente, durante su examen inicial, presión arterial sistólica $>140 \mathrm{mmHg}$ o diastólica > $90 \mathrm{mmHg}$. Con ayuda del diagnóstico pre-stroke, se consideró a los pacientes con diabetes mellitus cuando tenían una concentración de glucosa en suero $\geq$ $126 \mathrm{mg} / \mathrm{dL}$ o de glucosa en plasma $\geq 200 \mathrm{mg} / \mathrm{dL}$ dos horas después de la carga con $75 \mathrm{~g}$ glucosa oral. La hiperlipidemia se definió como el colesterol total $\geq$ $200 \mathrm{mg} / \mathrm{dL}$, o como lipoproteína de baja densidad $\geq$ $130 \mathrm{mg} / \mathrm{dL}$, o bien, si los pacientes estaban en terapia nutricional pre-stroke.

Se detectó fibrilación auricular y estenosis carotídea mediante diagnóstico por electrocardiografía de 
Cuadro I. Frecuencias de etiologías según CCS y características clínicas de los pacientes.

\begin{tabular}{lccccccc}
\hline & & $\begin{array}{c}\text { Edad } \\
\text { Media } \pm \text { DE }\end{array}$ & $\begin{array}{c}\text { Sexo } \\
\text { F\%/M\% }\end{array}$ & $\begin{array}{c}\text { HTA } \\
n(\%)\end{array}$ & $\begin{array}{c}\text { DBT } \\
n(\%)\end{array}$ & $\begin{array}{c}\text { DLP } \\
n(\%)\end{array}$ & $\begin{array}{c}\text { TBQ } \\
n(\%)\end{array}$ \\
\hline Aterosclerosis evidente & $8(8)$ & $56.5 \pm 15.9$ & $4 / 4$ & $4(4)$ & $2(2)$ & $1(1)$ & $3(3)$ \\
Aterosclerosis probable & $10(10)$ & $65 \pm 17.1$ & $3 / 7$ & $7(7)$ & $5(5)$ & $4(4)$ & $1(1)$ \\
Embolismo cardioaórtico evidente & $5(5)$ & $71.6 \pm 15.9$ & $1 / 4$ & $4(4)$ & $1(1)$ & $1(1)$ & $1(1)$ \\
Embolismo cardioaórtico posible & $16(16)$ & $44.5 \pm 13.3$ & $8 / 8$ & $7(7)$ & $5(5)$ & $6(6)$ & $3(3)$ \\
Oclusión de arterias pequeñas evidente & $13(13)$ & $66 \pm 18.9$ & $9 / 4$ & $5(5)$ & $1(1)$ & $3(3)$ & $3(3)$ \\
Oclusión de arterias pequeñas probable & $6(6)$ & $31.7 \pm 12.7$ & $1 / 5$ & $1(1)$ & $2(2)$ & $2(2)$ & $2(2)$ \\
$\begin{array}{l}\text { Otras causas evidentes/disección de } \\
\text { arteria aguda }\end{array}$ & $19(19)$ & $44 \pm 10.2$ & $13 / 8$ & $7(7)$ & $4(4)$ & $4(4)$ & $6(6)$ \\
$\begin{array}{l}\text { Otras causas probables/disección de } \\
\text { arteria aguda }\end{array}$ & $3(3)$ & $41.0 \pm 3.6$ & $7 / 2$ & 0 & $3(3)$ & $1(1)$ & $3(3)$ \\
$\begin{array}{l}\text { No determinado/no conocido/ } \\
\text { embólico criptogénico }\end{array}$ & $2(2)$ & $71.5 \pm 16.2$ & $0 / 2$ & 0 & 0 & 0 & 0 \\
$\begin{array}{l}\text { Etiología no determinada desconocida } \\
\text { Indeterminado/desconocido/otras }\end{array}$ & $3(3)$ & $45.7 \pm 9.8$ & $2 / 1$ & $1(1)$ & 0 & 0 & $1(1)$ \\
causas criptogénicas & $10(10)$ & $58 \pm 20.4$ & $3 / 7$ & $4(4)$ & 0 & 0 & $3(3)$ \\
$\begin{array}{l}\text { No determinado/no conocido/ } \\
\text { evaluación incompleta }\end{array}$ & $5(5)$ & $63 \pm 18.2$ & $3 / 2$ & $4(4)$ & $2(2)$ & $1(1)$ & $2(2)$ \\
\hline
\end{tabular}

Los números representan el tamaño de la muestra y entre paréntesis el porcentaje (n, \%). CCS = Causative Classification of Stroke System HTA = hipertensión arterial; DBT = diabetes mellitus, DLP = dislipidemias, TBQ = tabaquismo.

12 derivaciones y/o Holter e imagen vascular extracraneal, ya sea antes del ACVi y/o dentro de los seis primeros meses del evento. Se registraron, además, el hábito de fumar, la trombofilia y la enfermedad arterial periférica o coronaria asociadas con ACVi. El diagnóstico incluyó técnicas de imagen craneal y vascular, electrocardiografía de 12 derivaciones, ecocardiografía transtorácica y transesofágica, y monitorización con Holter de 24 horas. En el laboratorio central se determinaron en suero los niveles de los parámetros de coagulación básicos (KPTT, recuento de plaquetas y APP), marcadores de vasculitis (anticoagulantes lúpicos, anticuerpos anticardiolipinas, anticuerpos antinucleares, anti-ADN y anticuerpos antineutrófilos citoplasma), en su mayoría por ELISA y angiografía convencional, si fue necesario. La hiperhomocisteinemia circulante se determinó antes y después de la carga con metionina, lo que informó un valor medio de $11.04 \pm 3.7$ basal y una media de
$34.30 \pm 13.98$ en la postcarga (valores normales basal $<14$ y post < 35).

Un único neurólogo vascular, Juan José Martín, asignó a todos los pacientes un subtipo etiológico utilizando los sistemas de clasificación TOAST y CCS. La clasificación TOAST define cinco categorías: aterosclerosis de grandes vasos, oclusión arterial pequeña, cardioembolismo, otra etiología y accidente cerebrovascular de etiología indeterminada. Esta última categoría incluye casos con más de una causa competitiva, así como aquéllas en las que no se encuentra la, causa a pesar de la investigación adecuada, y también aquéIlos con evaluación incompleta. ${ }^{3}$ La clasificación CCS distribuye a los pacientes en cinco categorías basándose en la identificación de los múltiples potenciales mecanismos que conducirían al ACVi: cardioaórtica, la aterosclerosis de arterias grandes, oclusión de la arteria pequeña, otra causa y no determinados. La clasificación CCS asigna los subtipos evidente, probable 
y posible con base en las estimaciones de los riesgos de ACVi del paciente asociadas con patologías cardiacas vasculares, parámetros clínicos o de imagen que se conocen comúnmente; además, está vinculada a los mecanismos específicos de ACVi. ${ }^{2}$

Todos los pacientes cumplimentan la rutina de estudios establecida en el sanatorio.

Análisis estadístico: las proporciones independientes se compararon mediante la prueba de Fisher o $\chi^{2}$, según correspondía. Para el análisis de asociación se elaboraron tablas de contingencia. El acuerdo entre sistemas de clasificación se midió usando el estadístico K (kappa) con valores: $<0.20$ acuerdo pobre, 0.21-0.40 débil, 0.41-0.60 moderado, bueno (0.61 a 0.8 y y muy bueno $(0.81$ a 1$) .{ }^{8}$ Se utilizó la prueba de McNemar para comparar las probabilidades de clasificación discordantes, utilizando la corrección de Bonferroni para comparaciones múltiples. Se consideró significativo con $p$ valor menor de 0.05 con intervalo de confianza al $95 \%$. Los análisis se realizaron con el software InfoStat/P 2018.

\section{RESULTADOS}

El estudio incluyó a 100 pacientes, 42 (42\%) hombres y $58(58 \%)$ mujeres; la edad media fue de $52.97 \pm$ 11.77. El $51 \%$ de los pacientes fue hospitalizado en la institución y el resto fue reclutado del consultorio externo del Servicio de Neurología. Entre los factores de riesgo para $\mathrm{ACVi}$, 29\% de los pacientes fueron hiper- tensos, $23 \%$ presentaron dislipidemias y $23 \%$ diabetes.

Se considera al $55 \%$ de los pacientes como no fumador, $24 \%$ fumador y $13 \%$ exfumador (no se consideró la cantidad de cigarrillos que fumaban). A todos los pacientes se les practicó evaluación cardiológica mediante técnicas no invasivas y se observó que el $57 \%$ mostró datos clínicos normales, mientras que al $37 \%$ se le detectaron alteraciones patológicas; dentro de éstas, al 9\% de los casos se le detectó foramen oval permeable y al $10 \%$ arritmias (fibrilación auricular en mayor proporción, extrasístoles ventriculares y taquicardia paroxística supraventricular en menor cantidad). El 18\% de las anomalías restantes correspondieron a disecciones arteriales vertebrales, fibrosis septal y estenosis carotídeas con obstrucción luminal. Se diagnosticaron 16 pacientes con disección, 10 de ellos pertenecían a la categoría de CCS de otras causas evidentes/disección arterial agudo. De estos 10 sujetos, seis fueron femeninos con edad promedio de $38.10 \pm$ 10.28 (rango 22-52). El análisis multivariado para conocer si los factores de riesgo predisponentes para $\mathrm{ACVi}$ (edad, sexo, índice de masa corporal, tabaquismo, consumo de alcohol y/o drogas, hipertensión arterial, diabetes y dislipidemias) no arrojó ningún tipo de asociación, posiblemente debido al tamaño muestral.

Observamos que entre los pacientes que asistieron al Sanatorio Allende la etiología más frecuente, según el CCS, fue otras causas evidentes/disección arterial aguda con 19 pacientes (19\%), seguida de embolismo cardioaórtico posible con 16 pacientes (16\%) (Cuadro

Cuadro II. Frecuencias de etiologías según TOAST y características clínicas de los pacientes.

\begin{tabular}{|c|c|c|c|c|c|c|c|}
\hline Categoría & n (\%) & $\begin{array}{c}\text { Edad } \\
\text { Media } \pm \text { DE }\end{array}$ & $\begin{array}{c}\text { Sexo } \\
\text { F\%/M\% }\end{array}$ & $\begin{array}{l}\text { HTA } \\
\text { n (\%) }\end{array}$ & $\begin{array}{l}\text { DBT } \\
\text { n (\%) }\end{array}$ & $\begin{array}{l}\text { DLP } \\
\text { n (\%) }\end{array}$ & $\begin{array}{l}\text { TBQ } \\
\text { n (\%) }\end{array}$ \\
\hline $\begin{array}{l}\text { Aterosclerosis de } \\
\text { arterias grandes }\end{array}$ & $9(9)$ & $57 \pm 16.7$ & $2 / 7$ & $7(7)$ & $3(39)$ & $3(3)$ & $3(3)$ \\
\hline Cardioembolismo & $17(17)$ & $51.1 \pm 19.1$ & $6 / 11$ & $6(6)$ & $5(5)$ & $6(6)$ & $4(4)$ \\
\hline $\begin{array}{l}\text { Oclusión de arterias } \\
\text { pequeñas (lacunar) }\end{array}$ & $16(16)$ & $55.2 \pm 20.4$ & $8 / 8$ & $8(8)$ & $3(3)$ & $4(4)$ & $4(4)$ \\
\hline $\begin{array}{l}\text { ACVi agudo de otra } \\
\text { etiología }\end{array}$ & 19 (19) & $45.7 \pm 28$ & $11 / 8$ & $9(9)$ & $3(39)$ & $6(6)$ & $8(8)$ \\
\hline $\begin{array}{l}\text { ACVi de etiología } \\
\text { indeterminada }\end{array}$ & 39 (39) & $52.2 \pm 18.6$ & $21 / 18$ & $14(14)$ & $8(8)$ & $11(11)$ & $9(9)$ \\
\hline
\end{tabular}

Los números representan el tamaño de la muestra y entre paréntesis el porcentaje (n, \%). TOAST = Trial of Org 10172 in Acute Stroke Registry. HTA = hipertensión arterial; DBT = diabetes mellitus, DLP = dislipidemias, TBQ = tabaquismo. 
Cuadro III. Acuerdo diagnóstico entre CCS y TOAST.

\begin{tabular}{lcc}
\hline & Coeficiente K IC $_{95 \%}$ & Significación \\
\hline Aterosclerosis de grandes vasos & $1(1-1)$ & Muy bueno \\
Embolismo cardioaórtico & $0.964(0.896-1)$ & Muy bueno \\
Oclusión de arterias pequeñas & $0.978(0.986-1)$ & Muy bueno \\
Otras causas & $0.88(0.765-0.895)$ & Muy bueno \\
Causa indeterminada & $0.563(0.408-0792)$ & Moderado \\
Kappa media & $0.816(0.65-1.108)$ & Muy bueno \\
\hline
\end{tabular}

Los números representan el tamaño de la muestra y entre paréntesis el porcentaje (n, \%). CCS = Causative Classification of Stroke System. TOAST = Trial of Org 10172 in Acute Stroke Registry. K = coeficiente kappa de Cohen.

I). Según la clasificación CCS, tres pacientes (3\%) fueron diagnosticados con etiología no determinada desconocida, $10(10 \%)$ con etiología indeterminada desconocida/otras causas criptogénica, dos (2\%) son no determinada no conocida/embolica criptogénica y cinco (5\%) son de etiología no determinada no conocida/evaluación incompleta, dando un total de 20 (20\%) pacientes diagnosticados con causas indeterminadas.

Para el sistema TOAST, la etiología más frecuente correspondería a la categoría $\mathrm{V}, \mathrm{ACV}$ i de etiología indeterminada con 39 pacientes (39\%), y a la categoría IV, agudo de otra etiología con 19 pacientes (19\%). Las características demográficas y los factores de riesgos se muestran en el cuadro II. Aquellos pacientes a los que se les asignó oclusión de arterias pequeñas evidente fueron en su mayoría mujeres; en cambio, los categorizados como aterosclerosis probable fueron varones.

Se calculó el índice kappa de Cohen (k), estadístico que mide la concordancia o acuerdo entre las observaciones sobre un mismo caso con el objeto de identificar con qué sistema se arriba a un diagnóstico preciso. Los valores de kappa se calcularon para los cinco subtipos principales de ACVi comunes a ambos sistemas. El análisis de concordancia de etiologías reveló un kappa de 0.816 (intervalo de confianza [IC] del 95\%: 0.65 a 1.102) para los cinco subtipos. El acuerdo sobre subtipos específicos fue más alto para la aterosclerosis de arterias grandes $k=1$ (1-1) y más bajo para causas indeterminadas $(k=0.562$ [0.403-0.722]). El cuadro III proporciona los índices kappa de acuerdo medio de cada subtipo. Los dos sistemas identificaron diferente número de casos como indeterminados (CCS 20 casos y TOAST 39 casos). El acuerdo de TOAST y CCS para casos indeterminados fue sólo de $\mathrm{k}=0.562$
(IC del 95\%: 0.403 a 0.722). Los casos clasificados por CCS como "embolia criptogénica" o "sin clasificar" no tienen una categoría correspondiente en TOAST, por lo que los pacientes se incluyeron en la categoría "indeterminado". Una característica del sistema CCS es su capacidad de asignar pacientes con múltiples etiologías en los subtipos de otras causas.

\section{Discusión}

Por medio de nuestro estudio pudimos comparar la utilidad y confiabilidad de los sistemas de clasificaciones de etiologías del ACVi, CCS y TOAST. Observamos que con TOAST la categoría indeterminada representa el 39\% de la muestra; mientras que el CCS refinó la asignación en 16 subtipos y redujo el diagnóstico a 20 pacientes. Dado que Arsava y colaboradores demostraron que el diagnóstico por la clasificación CCS reduce el sesgo interobservador, no siendo igual para TOAST, ${ }^{9}$ se acordó como criterio que un solo neurólogo experto en enfermedades neurovasculares fuera el encargado de realizar el diagnóstico de ACVi por ambos sistemas.

Si bien hay poca información acerca de los tipos más frecuentes y metodología utilizada para la clasificación del ACVi en América Latina, un trabajo informó una alta frecuencia de hemorragias intracraneales que representaron del 26 al $46 \%$ de todos los ACVi. Entre los pacientes con infartos cerebrales, las lesiones ateroscleróticas intracraneales y la enfermedad de pequeños vasos fueron los mecanismos patógenos comunes subyacentes al ACVi. ${ }^{10}$ Rojas y colaboradores informaron en el 2006 que el subtipo, clasificado por TOAST, más frecuente en la Ciudad Autónoma de Buenos Aires fue la oclusión de arterias pequeñas, ${ }^{11}$ 
observación que coincidió con el estudio ReNACer; ${ }^{12}$ en nuestras observaciones este subtipo representó el 16\% según TOAST y el 19\% según CCS, pero en ningún caso fue la categoría más representativa; esto puede deberse a que el promedio de edad de los pacientes incorporados a nuestro estudio ronda los 50 años. En concordancia con nuestras observaciones para este grupo etario, Gokcal E describe la oclusión de arterias pequeñas como el subtipo más frecuente a etiología indeterminada, según TOAST y CCS. ${ }^{13}$ En nuestras observaciones el subtipo más frecuente es la etiología indeterminada cuando se utiliza la clasificación TOAST, con CCS observamos una distribución homogénea de los subtipos de ACVi.

Los niveles de concordancia informados por otros autores entre los sistemas de clasificación TOAST y CCS fueron altos, lo que coincide con nuestras observaciones. En un estudio prospectivo de cohortes del Reino Unido, un solo médico realizó la extracción y clasificación de datos en TOAST y CCS en 381 pacientes con primer ACVi. La concordancia entre los dos sistemas sobre subtipos específicos varió de muy bueno $(\mathrm{k}=$ 0.95 para cardioembolismo) a moderado $(\mathrm{k}=0.69)$ para otras causas indeterminadas. ${ }^{5}$ En el mismo estudio un centro en Inglaterra que incorporó 690 pacientes con ACVi informó muy buen acuerdo general con una kappa de concordancia similar al calculado por nuestro grupo $(\mathrm{k}=0.85) .{ }^{5}$ Nuestra evaluación mostró un acuerdo muy bueno en general, siendo moderado sólo para las causas indeterminadas. McArdle P y colaboradores resumen en un estudio multicéntrico, que incluye a 13,590 pacientes, que el acuerdo entre los diagnósticos de TOAST y CCS fue moderado; esto significa que los dos sistemas clasifican los casos de ACVi en diferentes categorías. ${ }^{5}$ La implicancia práctica de este hallazgo es que la combinación o comparación de clasificaciones a través de los sistemas debe realizarse con precaución y, cuando sea posible, se debe alentar a volver a analizar las variables clínicas antes de combinarlos. Estos autores recomiendan precaución cuando se comparan o combinan resultados basados en los dos sistemas. ${ }^{5}$

Recientemente, Bogiatzi y colaboradores proponen SPARKLE (Subtypes of Ischaemic Stroke Classification System) como un sistema de clasificación que tiene en cuenta la estenosis carotidea (carotid plaque burden) en la clasificación de arteriosclerosis de grandes vasos, aumentando la proporción de casos atribuibles a enfermedad de arterias grandes y que reduce la proporción clasificada como de etiología "indeterminada", resultando más eficaz en pacientes con accidente cerebro- vascular menor o ataque isquémico transitorio. ${ }^{13}$ Otro sistema de clasificación basado en arteriosclerosis de vasos pequeños, enfermedad cardiaca y otras causas (ASCO) podría contribuir a una mejor clasificación de los pacientes con $\mathrm{ACVi} .{ }^{14}$

Este trabajo presenta ciertas limitaciones, por ejemplo, el análisis retrospectivo no nos permitió incorporar a todos los pacientes llegados al servicio en el periodo estudiado, ya que no todos poseían los datos clínicos y bioquímicos completos para aplicar el algoritmo estandarizado. Si bien la mayoría de los pacientes poseía la información del ecocardiograma transesofágico y electrocardiograma, así como los parámetros plasmáticos de coagulación, otros estudios específicos se realizaron sólo cuando se indicaron clínicamente. La otra gran limitación de nuestro estudio es el pequeño tamaño muestral, principalmente porque representa a un solo centro médico.

En conclusión, nuestros resultados confirman una concordancia muy buena entre la clasificación del ACVi utilizando los sistemas TOAST y CCS. No hubo evidencia de que ninguno de los dos sistemas generara un exceso de sujetos clasificados en algún subtipo específico y no hubo evidencia de que el uso de CCS redujera significativamente la proporción de pacientes clasificados a subtipos indeterminados. Un nuevo estudio multicéntrico con un número mayor de pacientes, con un diseño prospectivo y estandarizado que los haga comparables entre sí sería de interés para corroborar estas observaciones.

Agradecimientos: los autores quieren agradecer a los pacientes y familiares de los pacientes por permitir utilizar los datos de las historias clínicas en este manuscrito.

Financiamiento: el presente trabajo fue financiado parcialmente por la Secretaría de Ciencia y Tecnología de la Universidad Nacional de Córdoba, Córdoba, Argentina.

\section{CONFLICTO DE INTERESES}

Los autores declaran no tener conflicto de intereses con la publicación de este manuscrito.

\section{Bibliografía}

1. Desai JA, Abuzinadah AR, Imoukhuede O, Bernbaum ML, Modi J, Demchuk AM et al. Etiologic classification of TIA and minor stroke by A-S-C-O and causative classification system 
as compared to TOAST reduces the proportion of patients categorized as cause undetermined. Cerebrovasc Dis. 2014; 38: 121-126.

2. Ay $H$, Benner $T$, Arsava $E M$, Furie $K L$, Singhal $A B$, Jensen $M B$ et al. A computerized algorithm for etiologic classification of ischemic stroke: The Causative Classification of Stroke System. Stroke. 2007; 38: 2979-2984.

3. Adams HP Jr, Bendixen BH, Kappelle LJ, Biller J, Love BB, Gordon DL et al. Classification of subtype of acute ischemic stroke. Definitions for use in a Stroke. multicenter clinical trial. TOAST. Trial of Org 10172 in Acute Stroke Treatment. Stroke. 1993; 24 (1): 35-41.

4. Lanfranconi S, Markus HS. Stroke subtyping for genetic association studies? A comparison of the CCS and TOAST classifications. Int J Stroke. 2013; 8: 626-631.

5. McArdle PF, Kittner SJ, Ay H, Brown RD Jr, Meschia JF, Rundek $T$ et al. Agreement between TOAST and CCS ischemic stroke classification: the NINDS SiGN study. Neurology. 2014; 28: 1653-1660.

6. Marnane M, Duggan CA, Sheehan OC, Merwick A, Hannon $\mathrm{N}$, Curtin D et al. Stroke subtype classification to mechanismspecific and undetermined categories by TOAST, A-S-C-O, and causative classification system: direct comparison in the North Dublin population stroke study. Stroke. 2010; 42: 1579-1586.

7. Powers WJ, Rabinstein AA, Ackerson T, Adeoye OP, Bambakidis NC, Becker K et al. 2018 Guidelines for the early management of patients with acute ischemic stroke: A Guideline for Healthcare Professionals from the American Heart Association/American Stroke Association. Stroke. 2018; 49: e46-e110.

8. López-de Ullibarri GI, Pita-Fernández S. Medidas de concordancia: el índice kappa en metodología de la investigación Unidad de Epidemiología Clínica y Bioestadística. Complexo Hospitalario Universitario de A Coruña (España). Cad Aten Primaria. 1999; 6: 169-171.

9. Arsava EM, Ballabio E, Benner T, Cole JW, Delgado-Martinez MP, Dichgans $M$ et al. The Causative classification of stroke system: an international reliability and optimization study. Neurology. 2010; 75: 1277-1284.

10. Saposnik G, Del Brutto $\mathrm{OH}$; Iberoamerican Society of Cerebrovascular Diseases. Stroke in South America: a systematic review of incidence, prevalence, and stroke subtypes. Stroke. 2003; 34: 2103-2107.

11. Rojas JI, Zurru MC, Patrucco L, Romano M, Riccio PM, Cristiano E. Registro de enfermedad cerebrovascular isquémica. Medicina (Buenos Aires). 2006; 66: 547-551.

12. Sposato LA, Esnaola MM, Zamora R, Zurrú MC, Fustinoni $O$, Saposnik G; ReNACer Investigators; Argentinian Neurological Society. Quality of ischemic stroke care in emerging countries: the Argentinian National Stroke Registry (ReNACer). Stroke. 2008; 39 (11): 3036-41.

13. Gokcal E, Niftallyev E, Asil T. Etiological classification of ischemic stroke in young patients: a comparative study of TOAST, CCS and ASCO. Acta Neurol Belg. 2017; 117: 643-648.

14. Bogiatzi C, Wannarong T, McLeod Al, Heisel M, Hackam D, Spence JD. SPARKLE (Subtypes of Ischaemic Stroke Classification System), incorporating measurement of carotid plaque burden: a new validated tool for the classification of ischemic stroke subtypes. Neuroepidemiology. 2014; 42: 243-251.

Correspondencia:

\section{Adela Sembaj}

Cátedra de Bioquímica y Biología Molecular.

Facultad de Ciencias Médicas,

Universidad Nacional de Córdoba, Argentina.

Segundo piso Pabellón Argentina,

Ciudad Universitaria, Córdoba.

Fax: 54- 351-4333024

E-mail: asembaj@biomed.uncor.edu. 